\title{
Establishment of an miR-137-knockout cell model using CRISPR/Cas9 genome editing
}

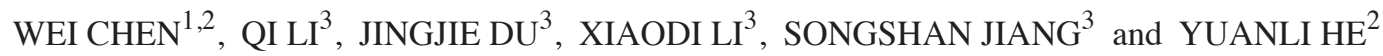 \\ ${ }^{1}$ Department of Gynecology, The Second Affiliated Hospital of Guangzhou Medical University, Guangzhou; \\ ${ }^{2}$ Department of Obstetrics and Gynecology, Zhujiang Hospital, Southern Medical University, \\ Guangzhou, Guangdong 510260; ${ }^{3}$ State Key Laboratory of Biocontrol, School of Life Sciences, \\ Sun Yat-sen University, Guangzhou, Guangdong 510006, P.R. China
}

Received November 1, 2016; Accepted November 2, 2017

DOI: $10.3892 / \mathrm{ol} .2018 .9096$

\begin{abstract}
MicroRNA-137 (miR-137) has been reported to be abnormally expressed in a variety of types of cancer, including ovarian cancer. However, the roles served by miR-137 in cancer are not fully understood. In the present study, 3 single guide RNAs (sgRNAs) were designed, synthesized and inserted into pXPR001 plasmids. The pXPR001-sgRNA plasmids were verified using sequencing and integrated into the genome of the ovarian cancer cell line, A2780, through lentiviral transduction, puromycin selection and single-cell culture. PCR products amplified from single-cell cultures using primers covering miR-137 targeting sites were sequenced to identify clones with miR-137 gene disruption. Genome editing was detected in $72 \%$ of the clones derived from sgRNA2, $4 \%$ from sgRNA3 and $0 \%$ from sgRNA1. Of the clones from sgRNA2, $32 \%$ contained 1 edited miR-137 allele and $40 \%$ contained 2 edited miR-137 alleles. The expression of miR-137 in clones \#2 and \#3 could not be detected by reverse transcriptionquantitative polymerase chain reaction. In addition, an MTT assay demonstrated that clones \#2 and \#3 exhibited enhanced proliferation. In conclusion, an miR-137-knockout cell model was successfully established in A2780 cells using CRISPR/Cas9 technology.
\end{abstract}

\section{Introduction}

MicroRNAs (miRNAs) are a class of small non-coding RNA of 18-24 nucleotides, which post-transcriptionally regulate the translation or degradation of protein-coding genes through imperfect base pairing with the 3'-untranslated region of

Correspondence to: Professor Yuanli He, Department of Obstetrics and Gynecology, Zhujiang Hospital, Southern Medical University, 253 Industrial Avenue, Guangzhou, Guangdong 510260, P.R. China

E-mail: heyuanli310@163.com

Key words: genome editing, CRISPR/Cas9, microRNA-137, cell model, A2780 cells target mRNAs (1). miRNAs are believed to be linked to tissue morphogenesis and cellular processes, e.g. apoptosis and major signaling pathways (2). For example, the miR-23a/24-2/27a cluster promotes cell invasion and metastasis by targeting Sprouty 2 in breast cancer (3). It is reported that $>60 \%$ of human protein-coding genes are regulated by miRNAs (4). As miRNAs may regulate the expression of tumor-associated genes in multiple tumor types, they are considered to be potential therapeutic targets $(5,6)$.

MicroRNA-137 (miR-137) is located on chromosome 1p22 and functions as a tumor suppressor by targeting a number of oncogenic mRNAs (7-10). Recent studies have demonstrated that miR-137 is downregulated in various types of tumor, suggesting that it can be regarded as a negative regulator of certain tumor-associated genes. For example, miR-137 acts as a tumor suppressor by directly targeting carboxyl-terminal binding protein 1 to inhibit the epithelial-mesenchymal transition and induce the apoptosis of melanoma cells (11). miR-137 inhibits the invasive ability of melanoma cells through the downregulation of melanogenesis associated transcription factor, enhancer of zeste 2 polycomb repressive complex 2 subunit, c-Met and Y-box binding protein 1 (12). To the best of our knowledge, only astrocyte elevated gene-1 has been reported as a target of miR-137 in human ovarian cancer (13). The function of miR-137 in ovarian cancer remains to be elucidated. The miR-137-knockout cell model established in the present study using a CRISPR/Cas9 editing approach will provide a useful platform for the functional study of ovarian cancer.

Clustered regularly interspaced short palindromic repeats (CRISPR) are a distinctive feature of the genomes of bacteria and archaea (14). Together with CRISPR-associated system (Cas) genes, they are hypothesized to be involved in resistance to bacteriophages, and resistance specificity is determined by spacer-phage sequence similarity (15). The strategy was first documented in 1987 when Ishino et al (16) were studying the isozyme conversion of alkaline phosphatase in Escherichia coli. The CRISPR/Cas9 system, consisting of Cas9 protein and guide RNA (gRNA), utilizes the type II prokaryotic CRISPR/Cas9 adaptive immune system and targets the Cas9 nuclease by a 20 nucleotide guide sequence cloned upstream of a 5'-NGG protospacer adjacent motif (17). 
Genome editing using CRISPR/Cas9 is hypothesized to be a more efficient strategy than designer nuclease technologies, including zinc-finger nucleases and transcription activator-like effectors, for the generation of genetically modified cells and organisms $(18,19)$. These easily engineered systems are increasingly being employed and have successfully knocked out or edited protein-coding genes in several model organisms and a variety of cell lines (20-25). Zhao et al (26) have reported the silencing of non-coding miRNA genes using CRISPR/Cas9. This suggested that miR-30a-5p and miR-21 could be edited in cells, and stimulated the production of the present study, in which an miR-137 gene knockout A2789 cell model was constructed using this technology. This may provide the basis for the functional study of miR-137 in ovarian cancer.

\section{Materials and methods}

Design of the sgRNAs. The sgRNAs targeting miR-137 were designed using CRISPR DESIGN (http://crispr.mit.edu/). A total of 320-bp sequences upstream of 5'-NGG were chosen as sgRNAs, and BsmBIsticky ends were attached to flank the sgRNAs. The pairs of oligonucleotides designed for the miR-137 site sequences (Fig. 1A) were sgRNA1, forward, 5'-CAC CGCCTCTGACTCTCTTCGGTGA-3' and reverse, 5'-AAA CTCACCGAAGAGAGTCAGAGGC-3'; sgRNA2, forward, 5'-CACCGCTTAAGAATACGCGTAGTC-3' and reverse, 5'-AAACCGACTACGCGTATTCTTAAGC-3'; sgRNA3, forward, 5'-CACCGCTAAACGCCCGTGGTTGCCA-3', and reverse, 5'-AAACTGGCAACCACGGGCGTTTAGC-3'.

Construction of sgRNA expression vectors. Each oligonucleotide was diluted to $10 \mu \mathrm{M}$, and each pair of oligonucleotides were mixed in equal volumes. Next, $1 \mu \mathrm{l}$ combined oligonucleotides, $1 \mu 1$ annealing buffer and $6 \mu 1$ water were mixed in a total reaction volume of $8 \mu \mathrm{l}$, placed in boiling water for $10 \mathrm{~min}$ and then decreased to $25^{\circ} \mathrm{C}$. The annealed oligonucleotides were added $1 \mu \mathrm{l}$ ligation buffer and ligated with BsmB1-digested linearized pXPR001 plasmids $(1 \mu \mathrm{l})$. The ligation mix was transformed into competent Dh5 $\alpha$ E. coli cells (Shanghai DingGuo Biotech., Co., Ltd., Shanghai, China) and the constructs were confirmed by sequencing.

Cell lines and cell culture. 293T and A2780 cells from the American Type Culture Collection (Manassas, VA, USA) were cultured in Dulbecco's modified Eagle's medium or RPMI, respectively, supplemented with $10 \%$ fetal bovine serum and penicillin/streptomycin (100 U/ml; Invitrogen; Thermo Fisher Scientific, Inc., Waltham, MA, USA), and maintained in an incubator at $37^{\circ} \mathrm{C}$ with $5 \% \mathrm{CO}_{2}$.

Virus generation and infection. 293T cells $\left(8 \times 10^{5}\right)$ were seeded in a $6-\mathrm{cm}$ plate 1day prior to transfection. Co-transfection of the $1.8 \mu \mathrm{g}$ pPAX2 packaging plasmid, $0.8 \mu \mathrm{g}$ pMD2.G envelope plasmid and $2.5 \mu \mathrm{g}$ pXPR001-sgRNA vector was performed using Fugene HD transfection reagent (Roche Diagnostics, Basel, Switzerland). A total of $48 \mathrm{~h}$ after transfection, viral supernatants were harvested and stored at $-80^{\circ} \mathrm{C}$.

Single-cell culture and selection of miR-137-knockout clones. A2780 cells were seeded in a 6 -well plate at $4 \times 10^{5}$ cells/well and $100 \mu \mathrm{l}$ viral supernatants were added $24 \mathrm{~h}$ later. Puromycin (800 ng/ml)was added 1 day after transduction for a 2-day period of selection. The remaining cells were sorted into 96-well plates by flow cytometry for single-cell culture. When the cells reached a certain density, they were then transferred to 24-well plates. In total, 500 cells were used for direct identification using PCR. These cells were directly added to $25 \mu \mathrm{l}$ PCR system. The PCR system consisted of $12.5 \mu 12 \mathrm{X}$ PCR Buffer for KOD FX, $5 \mu 12 \mathrm{~mm}$ dNTP, $1.5 \mu 1$ positive and $5 \mu \mathrm{M}$ reverse primers, $2 \mu \mathrm{l}$ PCR template cells and $4 \mu \mathrm{l}$ $\mathrm{ddH}_{2} \mathrm{O}$. Polymerase chain reaction (PCR) was performed using KOD FX DNA polymerase (Toyobo Life Science, Osaka, Japan), using primers covering the 3 miR-137 targeting sites to identify the cell clones with miR-137 gene disruption. The primer sequences used were as follows: Forward, 5'-TGG ATCCTTCTTTAGGGAAATCGAGT-3', reverse, 5'-GAA AACACCCGAGGAAATGAAAAGAAC-3'. Thermocycling took place as follows: Denaturation at $95^{\circ} \mathrm{C}$ for $5 \mathrm{~min}$, followed by 35 cycles of denaturation at $98^{\circ} \mathrm{C}$ for $10 \mathrm{sec}$, annealing and extension at $68^{\circ} \mathrm{C}$ for $40 \mathrm{sec}$. The PCR products were separated in a $1.5 \%$ agarose gel, and were extracted from the gel using a gel purification kit (Omega Bio-Tek, Inc., Norcross, GA, USA). The sequence of the sgRNA2 construct is illustrated in Fig. 1B.

RNA extraction \& reverse transcription-quantitative (RT-q) $P C R$. Total RNA was extracted from the cells with TRIzol (Invitrogen; Thermo Fisher Scientific, Inc.) and was reverse transcribed by ReverTraAce- $\alpha$-Transcriptase (Toyobo Life Science), according to the manufacturers' protocol. The expression of miR-137 in cell lines was quantified using a SYBR ${ }^{\circledR}$ Premix Ex Taq ${ }^{\mathrm{TM}}$ II (TliRNaseH Plus) kit (Takara Bio, Inc., Otsu, Japan); U6 was used as an internal control. Thermocycling was performed using a Light Cycler 480 Real-Time PCR system (Roche Diagnostics, Basel, Switzerland) with the following conditions: Denaturation at $95^{\circ} \mathrm{C}$ for $5 \mathrm{~min}$, followed by 40 cycles of denaturation at $95^{\circ} \mathrm{C}$ for $10 \mathrm{sec}$, annealing at $60^{\circ} \mathrm{C}$ for $20 \mathrm{sec}$, and extension at $72^{\circ} \mathrm{C}$ for $30 \mathrm{sec}$. The data were analyzed using LightCycler 480 software version 1.5 (Roche Diagnostics, Basel, Switzerland). The relative fold changes of miR-137 expression in the miR-137-knockout cells vs. control cells were calculated using the $2^{-\Delta \Delta \mathrm{Cq}}$ method (27). The primers used were as follows: miR-137RT: 5'-CTCAAC TGGTGTCGTGGAGTCGGCAATTCAGTTGAGCTACGC GT-3', miR-137 forward, 5'-CCAGCTGGGTTAAGAATA CGCGTAG-3', miR-137 reverse, 5'-CTGGTGTCGTGGAGT CGGCAATT-3'; U6 RT, 5'-CTCAACTGGTGTCGTGGA GTCGGCAATTCAGTTGAGAAAAATATG-3', U6 forward, 5'-CTCGCTTCGGCAGCACA-3', U6 reverse, 5'-AACGCT TCACGAATTTGCGT-3'.

MTT assay. A2780 cells and miR-137-knockout single-cell clones derived from A2780 cells were seeded in a 96-well plate at $1 \times 10^{4}$ cells/well. At $72 \mathrm{~h}, 5 \mathrm{mg} / \mathrm{ml}$ MTT was added to the medium, and the plate was incubated in a $37^{\circ} \mathrm{C}$, $5 \% \mathrm{CO}_{2}$ incubator for $4 \mathrm{~h}$. DMSO was added at a volume of $100 \mathrm{ml} /$ well, and the plate was thoroughly mixed for $10 \mathrm{~min}$. The absorbance of the samples was measured at $490 \mathrm{~nm}$ by a microplate reader (BioTek Instruments, Inc., Winooski, VT, USA). 
A
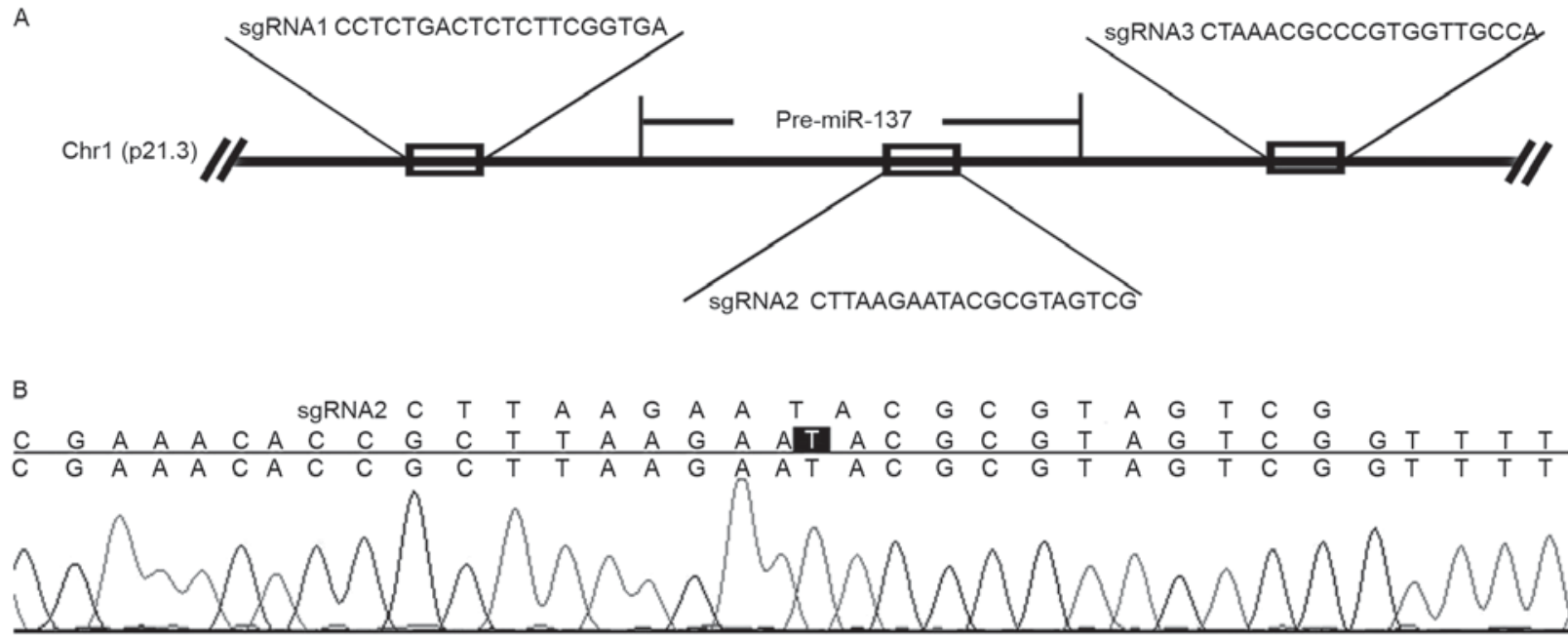

Figure 1. Design and cloning of 3 sgRNAs. (A) Corresponding DNA sequence of 3 sgRNAs and their locations relative to pre-miR-137. (B) Sequence of the sgRNA2 construct. sgRNA, single guide RNA; miR, microRNA.

A

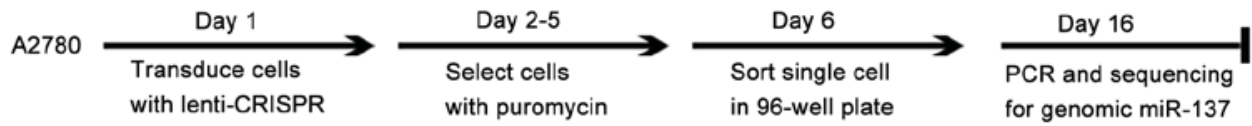

B

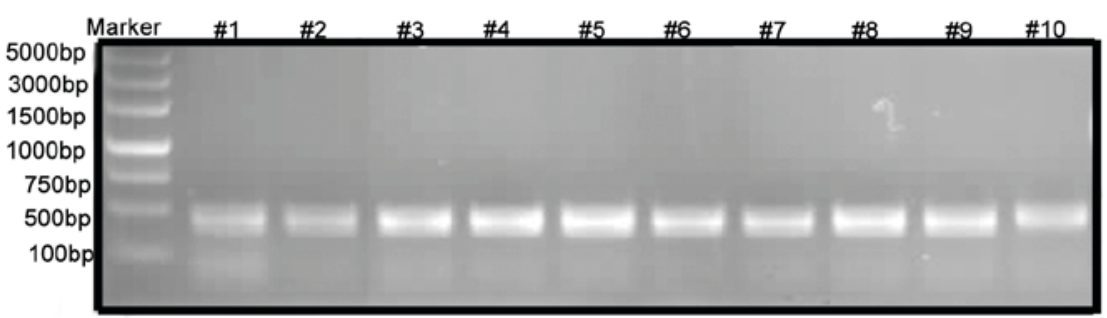

Figure 2. Selection of single-cell culture in A2780 cells. (A) The process of miR-137-knockout in A2780 cells using CRISPR/Cas9 technology. (B) PCR products amplified from single-cell cultures of single guide RNA2. PCR, polymerase chain reaction; miR, microRNA.

Statistical analysis. GraphPad Prism (version 5.0; GraphPad Software, Inc., La Jolla, CA, USA) was used for data analysis. The data are presented as the mean \pm standard deviation of $\geq 3$ separate experiments and were analyzed using one-way analysis of variance and Dunnett's post-hoc test). $\mathrm{P}<0.05$ was considered to indicate a statistically significant difference. $\mathrm{P}<0.05,0.01,0.001$ or 0.0001 are indicated by asterisks ${ }^{*},{ }^{* *},{ }^{* * *}$, or ${ }^{* * * *}$, respectively.

\section{Results}

Selection of the single-cell cultures with miR-137-knockout. The expression level of miR-137 in A2780 was the highest in a panel of 7 ovarian cancer cell lines (data not shown). Therefore, A2780 cells were selected for the establishment of the miR-137-knockout cell model. Single-cell cultures exhibitingmiR-137-knockout were selected according to the process illustrated in Fig. 2A. The transduced cells were selected in $800 \mathrm{nM}$ puromycin for 2 days, and the single-cell cultures with miR-137 disruption were identified via sequencing the PCR products (Fig. 2B) amplified from single-cell cultures using primers extending across the 3 miR-137-targeting sites.

A total of 25 single-cell cultures transduced with each sgRNA were selected for miR-137-knockout identification. None of the sgRNA1 cultures were positive for miR-137-knockout; however, 18/25 single-cell cultures of sgRNA2 and 1/25 single-cell cultures of sgRNA3 were positive for miR-137-knockout. This demonstrated a higher gene knockout efficiency of sgRNA2. Further analysis demonstrated that $32 \%$ of the sgRNA2 single-cell cultures had 1allele of miR-137 edited, and $40 \%$ had 2alleles edited. For example, in the target site region of clone \#2, a 2-allele knockout single-cell culture, there were 17 and 7-base deletions for each allele. In the target site region of clone \#3, another 2-allele knockout single-cell culture, there were 8-base deletions for both alleles (Fig. 3A). For comparison, the sequencing result of a miR-137-knockout single-cell culture of sgRNA3 is included in Fig. 3B, which did not disrupt the pre-miR-137 sequence. Therefore, clones $\# 2$ and \#3 derived from sgRNA2 were selected for further experiments. 


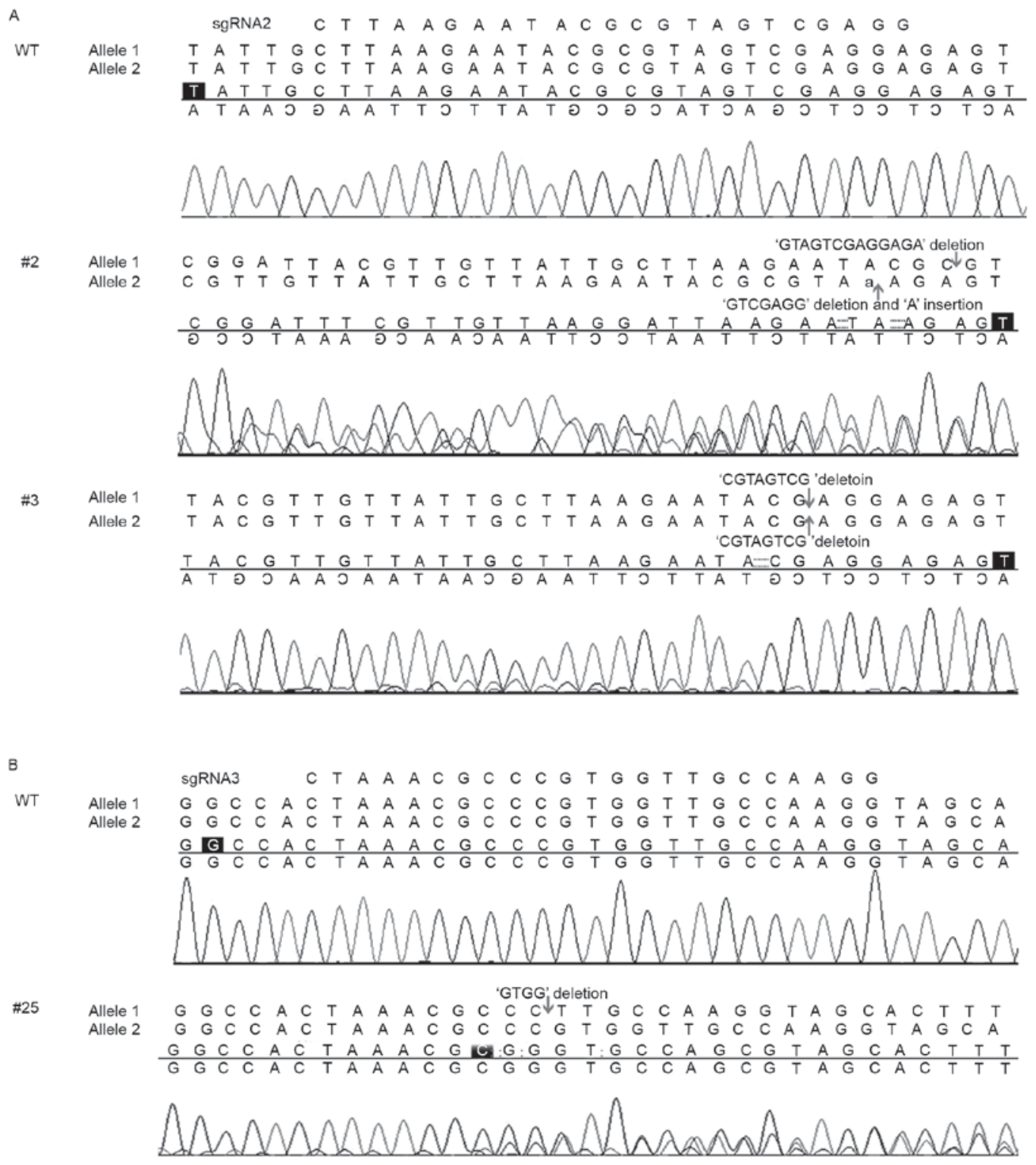

Figure 3. Chromatograms of PCR products amplified from the cell clones with miR-137 disruption. (A) Sequencing results of clones \#2 and \#3 derived from sgRNA2. (B) Sequencing of clone \#25 derived from sgRNA3. The reverse and forward PCR primers were used as sequencing primers for (A) and (B), respectively. The deleted or inserted sequences are indicated by arrows. PCR, polymerase chain reaction; miR, microRNA; WT, wild-type.

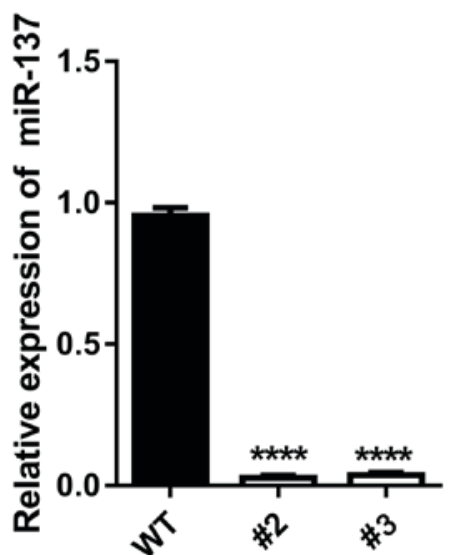

Figure 4. Expression of miR-137 in clones \#2 and \#3 and A2780 cells expressing the empty vector was detected by reverse transcription-quantitative polymerase chain reaction. U6 was used as an internal control. ${ }^{* * * * *} \mathrm{P}<0.0001$ vs. the control. miR, microRNA; WT, wild type (empty vector).

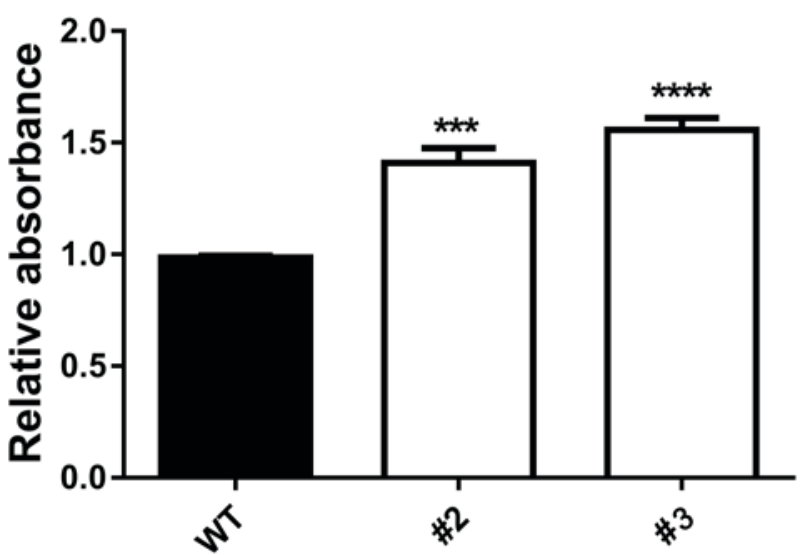

Figure 5. miR-137-knockout promotes cell proliferation in A2780 cells. The growth of A2780 cells expressing empty vector (WT) or miR-137-knockout clones (\#2 and \#3) were assessed using MTT assays at $72 \mathrm{~h}$. Each data point was measured in triplicate. The values represent the mean \pm standard deviation. ${ }^{* * *} \mathrm{P}<0.001$ and ${ }^{* * * * *} \mathrm{P}<0.0001$ vs. the control. miR, microRNA; WT, wild-type. 
Establishment of the miR-137-knockout cell model. RT-qPCR was performed to detect the expression levels of miR-137 in the miR-137-knockout clones. The RT-qPCR results of clones \#2 and \#3 indicated the low expression levels of miR-137 compared with wild-type cells $(\mathrm{P}<0.0001$; Fig. 4). This suggested that the cell model with miR-137-knockout had been successfully established using CRISPR/Cas9 technology.

miR-137-knockout promotes proliferation in A2780 cells. To investigate the effect of miR-137-knockout in A2780 ovarian cancer cells, the rate of proliferation was assessed using an MTT assay. A total of $72 \mathrm{~h}$ after seeding, the relative absorbance values of the miR-137-knockout clones (\#2 and \#3) were significantly increased compared with A2780 cells expressing the CRISPR/cas9 empty vector (WT; $\mathrm{P}<0.001$ for \#2 and $\mathrm{P}<0.0001$ for \#3; Fig. 5). These results suggest that the miR-137-knockout promoted the proliferation of A2780 cells.

\section{Discussion}

Precise and efficient genome-targeting technologies allowing selective perturbation of individual genetic elements can facilitate the reverse engineering or reconstruction of useful biological systems. Although genome-editing technologies, including designer zinc fingers and transcription activator-like effectors, have begun to enable targeted genome modifications (19), each of these platforms has limitations (28). Genome editing using CRISPR/Cas9 is a more affordable and efficient strategy for the generation of gene-modified cells and organisms. It is now the most popular tool for the precise alteration of the genomes of diverse species. Its application in genome-wide studies will enable large-scale screening for drug targets and enhance the development of human gene therapy (29).

Mali et al (17) obtained targeting rates of $10-25 \%$ of the endogenous AAVS1 locus in 293T cells, 13-38\% in K562 cells, and $2-4 \%$ in induced pluripotent stem cells. In the present study, miR-137 gene knockout efficiency was $56 \%$ for sgRNA 2 and $2 \%$ for sgRNA3, whereas sgRNA1 demonstrated no editing efficiency, indicating that differences in target sequences affected the efficiency of knockout.

miRNAs can regulate gene expression in diverse biological processes, including immune modulation, metabolic control, neuronal development, cell cycle, muscle differentiation and stem cell differentiation (2). Previous studies have indicated that miR-137 serves critical roles in gastric cancer, glioblastoma, non-small cell lung cancer, colorectal cancer, ovarian cancer and neuroblastoma (9,25-29). In the present study, sgRNAs were designed and synthesized to target miR-137 in A2780 cells. The delivering Cas9 nuclease and miR-137-specific sgRNAs were transformed into lentiviruses, which were used to infect the A2780 cells. These cells were selected with puromycin and sorted into 96-well plates for single-cell culture. The RT-qPCR results demonstrated that miR-137 was minimally detected in the miR-137-knockout cells. An MTT assay indicated that miR-137-knockout promoted proliferation in A2780 cells. Thus, the miR-137-knockout A2780 cell model was successfully established using CRISPR/Cas9 technology and exhibited a functional effect.

\section{Acknowledgements}

The present study was supported by funding from the Health and Family Planning Commission of Guangdong Province (grant no., A2015314).

\section{Competing interests}

The authors declare that they have no competing interests.

\section{References}

1. Bartel DP: MicroRNAs: Genomics, biogenesis, mechanism, and function. Cell 116: 281-297, 2004.

2. Kloosterman WP and Plasterk RH: The diverse functions of microRNAs in animal development and disease. Dev Cell 11: 441-450, 2006

3. Li X, Liu X, Xu W, Zhou P, Gao P, Jiang S, Lobie PE and Zhu T: c-MYC-regulated miR-23a/24-2/27a cluster promotes mammary carcinoma cell invasion and hepatic metastasis by targeting Sprouty2. J Biol Chem 288: 18121-18133, 2013.

4. Friedman RC, Farh KK, Burge CB and Bartel DP: Most mammalian mRNAs are conserved targets of microRNAs. Genome Res 19: 92-105, 2009.

5. Volinia S, Calin GA, Liu CG, Ambs S, Cimmino A, Petrocca F, Visone R, Iorio M, Roldo C, Ferracin M, et al: A microRNA expression signature of human solid tumors defines cancer gene targets. Proc Natl Acad Sci USA 103: 2257-2261, 2006.

6. Christopher AF, Kaur RP, Kaur G, Kaur A, Gupta V and Bansal P: MicroRNA therapeutics: Discovering novel targets and developing specific therapy. Perspect Clin Res 7: 68-74, 2016.

7. Feng Q, Wu Q, Liu X, Xiong Y and Li H: MicroRNA-137 acts as a tumor suppressor in osteosarcoma by targeting enhancer of zeste homolog 2. Exp Ther Med 13: 3167-3174, 2017.

8. Li X, Chen W, Zeng W, Wan C, Duan S and Jiang S: microRNA-137 promotes apoptosis in ovarian cancer cells via the regulation of XIAP. Br J Cancer 116: 66-76, 2017.

9. Sun L, Liang J, Wang Q, Li Z, Du Y and Xu X: MicroRNA-137 suppresses tongue squamous carcinoma cell proliferation, migration and invasion. Cell Prolif 49: 628-635, 2016.

10. Wu L, Chen J, Ding C, Wei S, Zhu Y, Yang W, Zhang X, Wei X and Han D: MicroRNA-137 contributes to dampened tumorigenesis in human gastric cancer by targeting AKT2. PLoS One 10: e0130124, 2015.

11. Deng Y, Deng H, Bi F, Liu J, Bemis LT, Norris D, Wang XJ and Zhang Q: MicroRNA-137 targets carboxyl-terminal binding protein 1 in melanoma cell lines. Int J Biol Sci 7: 133-137, 2011.

12. Luo C, Tetteh PW, Merz PR, Dickes E, Abukiwan A Hotz-Wagenblatt A, Holland-Cunz S, Sinnberg T, Schittek B, Schadendorf D, et al: miR-137 inhibits the invasion of melanoma cells through downregulation of multiple oncogenic target genes. J Invest Dermatol 133: 768-775, 2013.

13. Guo J, Xia B, Meng F and Lou G: miR-137 suppresses cell growth in ovarian cancer by targeting AEG-1. Biochem Biophys Res Commun 441: 357-363, 2013.

14. Jansen R, Embden JD, Gaastra W and Schouls LM: Identification of genes that are associated with DNA repeats in prokaryotes. Mol Microbiol 43: 1565-1575, 2002.

15. Barrangou R, Fremaux C, Deveau H, Richards M, Boyaval P, Moineau S, Romero DA and Horvath P: CRISPR provides acquired resistance against viruses in prokaryotes. Science 315: 1709-1712, 2007.

16. Ishino Y, Shinagawa H, Makino K, Amemura M and Nakata A: Nucleotide sequence of the iap gene, responsible for alkaline phosphatase isozyme conversion in Escherichia coli, and identification of the gene product. J Bacteriol 169: 5429-5433, 1987.

17. Mali P, Yang L, Esvelt KM, Aach J, Guell M, DiCarlo JE, Norville JE and Church GM: RNA-guided human genome engineering via Cas9. Science 339: 823-826, 2013.

18. Cong L, Ran FA, Cox D, Lin S, Barretto R, Habib N, Hsu PD, Wu X, Jiang W, Marraffini LA and Zhang F: Multiplex genome engineer using CRISPR/Cas systems. Science 339: 819-823, 2013. 
19. LaFountaine JS, Fathe K and Smyth HD: Delivery and therapeutic applications of gene editing technologies ZFNs, TALENs, and CRISPR/Cas9. Int J Pharm 494: 180-194, 2015.

20. Sakuma T, Nishikawa A, Kume S, Chayama K and Yamamoto T: Multiplex genome engineering in human cells using all-in-one CRISPR/Cas9 vector system. Sci Rep 4: 5400, 2014.

21. Schwank G, Koo BK, Sasselli V, Dekkers JF, Heo I, Demircan T, Sasaki N, Boymans S, Cuppen E, van der Ent CK, et al: Functional repair of CFTR by CRISPR/Cas9 in intestinal stem cell organoids of cystic fibrosis patients. Cell Stem Cell 13: 653-658, 2013.

22. Wu Y, Liang D, Wang Y, Bai M, Tang W, Bao S, Yan Z, Li D and Li J: Correction of a genetic disease in mouse via use of CRISPR-Cas9. Cell Stem Cell 13: 659-662, 2013.

23. Hruscha A, Krawitz P, Rechenberg A, Heinrich V, Hecht J, Haass C and Schmid B: Efficient CRISPR/Cas9 genome editing with low off-target effects in zebrafish. Development 140: 4982-4987, 2013
24. Friedland AE, Tzur YB, Esvelt KM, Colaiacovo MP, Church GM and Calarco JA: Heritable genome editing in C. Elegans via a CRISPR-Cas9 system. Nat Methods 10: 741-743, 2013.

25. Yu Z, Ren M, Wang Z, Zhang B, Rong YS, Jiao R and Gao G: Highly efficient genome modifications mediated by CRISPR/Cas9 in Drosophila. Genetics 195: 289-291, 2013.

26. Zhao Y, Dai Z, Liang Y, Yin M, Ma K, He M, Ouyang H and Teng CB: Sequence-specific inhibition of microRNA via CRISPR/CRISPRi system. Sci Rep 4: 3943, 2014.

27. Livak KJ and Schmittgen TD: Analysis of relative gene expression data using real-time quantitative PCR and the 2(-Delta Delta C(T)) method. Methods 25: 402-408, 2001.

28. Hsu PD, Lander ES and Zhang F: Development and applications of CRISPR-Cas9 for genome engineering. Cell 157: 1262-1278, 2014.

29. Doudna JA and Charpentier E: Genome editing. The new frontier of genome engineering with CRISPR-Cas9. Science 346: 9, 2014. 